\title{
Solution of Radial Schrödinger Equation with Yukawa Potential Using Bethe Ansatz Method
}

\author{
M. Mohammadi SABeT* \\ Department of Physics, Faculty of Science, Ilam University, Ilam, Iran
}

Received: 11.05.2021 \& Accepted: 01.06.2021

Doi: 10.12693/APhysPolA.140.97

*e-mail: m.mohamadisabet@ilam.ac.ir

\begin{abstract}
The quasi-exact Bethe ansatz method has been examined for solving the radial Schrödinger equation with the Yukawa potential as an important central interaction for computing bound states of neutral atoms. The method, in spite of its simplicity with respect to other methods, leads to the ground state and low excited states of the radial Schrödinger equation with a good accuracy. It is found that the results are generally in agreement with the results of others and, therefore, this method can be used for solving the Schrödinger equation instead of other numerical and analytical methods. It was also found that the energy eigenvalues reach that of the Coulomb potential in the limit of zero screening parameter. The method has been briefly generalized to arbitrary dimension for the Yukawa potential inspiring the 3-dimensional trail wave function.
\end{abstract}

topics: Yukawa potential, Bethe ansatz method, quasi-exact solution, Schrödinger equation

\section{Introduction}

In order to find the properties of quantum mechanical systems, one needs to solve a relativistic or non-relativistic wave equation. Solving these equations leads to energy spectra and wave functions of the system needed to find other physical properties. Unfortunately, the wave equation can be solved exactly only for some potentials and, therefore, in recent years different work has been carried out to find the exact solutions of these equations with different potentials [1-16].

Since analytical methods to find exact solutions are not as simple as expected, different mathematical methods have been used to approximate the solutions of quantum systems, including supersymmetry quantum mechanics [17, 18], group theoretical approach $[19,20]$ and the Nikiforov-Uvarov (NU) method [21].

Other methods for solving wave equations, known as quasi-exact solutions, find the polynomial solutions [22-24], one of which is the Bethe ansatz method (BAM) [25]. This method finds the ground state, as well as excited states of systems approximately. BAM works straightforwardly and is based on finding a polynomial solution of a second order differential equation. In this method, there is a set of $n$ equations to find some distinct real roots and the solution is the product of the factors of these roots.
On the other hand, one of the most important potentials in physics is the Yukawa potential [26], which is usually used to calculate the bound state of neutral atoms [27-29]. This potential, known as the Debye-Hückel (Thomas-Fermi) potential in plasma (solid state physics), has the following form:

$$
V(r)=-V_{0} \frac{\mathrm{e}^{-\alpha r}}{r},
$$

where $\alpha$ (screening parameter) and $V_{0}$ (strength of potential) take different values depending on the problem under investigation. The Yukawa potential is not shape-invariant and thus an exact analytical solution is not possible. Hence, different methods have been used to solve the wave equation with the Yukawa potential. Greene and Aldrich [30] generalized the variational wave function, used by Lam and Varshni for $s$-states [31], to $l \neq 0$ states. They used the effective Hulthén potential as an approximation for the Yukawa potential and calculated the energy of $2 p, 3 p, 3 d$, etc. levels. Gönül et al. [32] used the supersymmetry framework and the Riccuti equation to solve the Schrödinger equation and find the bound states of the Yukawa potential. This method is based on separating the Schrödinger equation into two parts, one of which has an exact solution and the other leads to approximated treatment. Other methods are the asymptotic iteration method [33], the quasi-linearization method [34], the numerical method [35, 36], NU [37], and the canonical transformations of the Schrödinger equation [38]. 
Based on the above discussion, in this paper BAM has been used to find the energy eigenstates and eigenvalues of the Yukawa potential in a nonrelativistic quantum mechanical (the Schrödinger equation) system. Therefore, the structure of this paper is as follows: firstly, the BAM method is presented in Sect. 2. Then, this method is used for the Yukawa potential and the results are presented as well as some comparisons are made. Further, a brief discussion on generalization to $N$ dimensions is given in Sect. 4. Finally, the conclusion follows.

\section{Bethe ansatz method}

Consider the following second order differential equation:

$$
\left(P(z) \frac{\mathrm{d}^{2}}{\mathrm{~d} z^{2}}+\frac{\mathrm{d}}{\mathrm{d} z} Q(z)+W(z)\right) S(z)=0,
$$

where $P(z), Q(z)$ and $W(z)$ are the polynomials of degree 4,3 , and 2 with constant coefficients $a_{k}, b_{k}$, and $c_{k}$, respectively

$$
\begin{aligned}
& P(z)=\sum_{k=0}^{4} a_{k} z^{k}, \\
& Q(z)=\sum_{k=0}^{3} b_{k} z^{k}, \\
& W(z)=\sum_{k=0}^{2} c_{k} z^{k} .
\end{aligned}
$$

Following the steps suggested by Zhong [25], we look for the polynomial $S(z)$ of degree $n$ as follows:

$$
\begin{aligned}
& S(z)=\prod_{i=1}^{n}\left(z-z_{i}\right), \\
& S(z)=1 \quad \text { for } n=0,
\end{aligned}
$$

where $z_{i}$ are the distinct real roots of $S(z)$ given by a set of $n$ algebraic equations to determine these roots,

$$
\begin{aligned}
& c_{2}=-n(n-1) a_{4}-n b_{3}, \\
& c_{1}=-\left(2(n-1) a_{4}+b_{3}\right) \sum_{i=1}^{n} z_{i}-n(n-1) a_{3}-n b_{2}, \\
& c_{0}=-\left(2(n-1) a_{4}+b_{3}\right) \sum_{i=1}^{n} z_{i}^{2}-2 a_{4} \sum_{i<j}^{n} z_{i} z_{j} \\
&-\left(2(n-1) a_{3}+b_{2}\right) \sum_{i=1}^{n} z_{i}-n(n-1) a_{2}-n b_{1} .
\end{aligned}
$$

These roots satisfy the Bethe ansatz equations

$$
\begin{aligned}
& \sum_{j \neq i}^{n} \frac{2}{z_{i}-z_{j}}+\frac{b_{3} z_{i}^{3}+b_{2} z_{i}^{2}+b_{1} z_{i}+b_{0}}{a_{4} z_{i}^{4}+a_{3} z_{i}^{3}+a_{2} z_{i}^{2}+a_{1} z_{i}+a_{0}}=0 \\
& \quad i=1,2, \ldots, n .
\end{aligned}
$$

Equations (5)-(8) give all polynomials $W(z)$ in such a way that $S(z)$ is the polynomial solution of the differential (2).

\section{Quasi-exact solution of radial Schrödinger equation with Yukawa potential}

In order to investigate quantum mechanical systems with a central potential $(V(r))$, one should solve the Schrödinger equation which is as follows:

$$
\left(-\frac{\hbar^{2}}{2 m} \nabla^{2}+V(r)\right) \psi(r, \theta, \phi)=E \psi(r, \theta, \phi),
$$

where $m$ is the mass of a particle, $\hbar$ is the reduced Planck constant and $r, \theta$, and $\phi$ are the spherical coordinates. The wave function $\psi(r, \theta, \phi)$ is characterized by quantum numbers $n, l, m_{l}$. Now, considering $V(r)$ as the Yukawa potential (1) and using the separation of variables as $\psi(r, \theta, \phi)=$ $\frac{R_{n l}(r)}{r} Y_{l m_{l}}(\theta, \phi)$, the following differential equation is obtained for the radial Schrödinger equation $(\hbar=1)$ :

$\left[\frac{\mathrm{d}^{2}}{\mathrm{~d} r^{2}}+2 m\left(E+V_{0} \frac{\mathrm{e}^{-\alpha r}}{r}\right)-\frac{l(l+1)}{r^{2}}\right] R_{n l}(r)=0$.

In order to solve this equation, the following approximation is used [37]:

$$
\frac{1}{r^{2}} \approx 4 \alpha^{2} \frac{\mathrm{e}^{-2 \alpha r}}{\left(1-\mathrm{e}^{-2 \alpha r}\right)^{2}} .
$$

Therefore, we have

$$
\frac{1}{r} \approx 2 \alpha \frac{\mathrm{e}^{-\alpha r}}{\left(1-\mathrm{e}^{-2 \alpha r}\right)},
$$

and the Yukawa potential reduces to

$$
V(r) \approx-2 \alpha V_{0} \frac{\mathrm{e}^{-\alpha r}}{\left(1-\mathrm{e}^{-2 \alpha r}\right)} .
$$

The accuracy of this approximation for the Yukawa potential (13) has been investigated in [37]. The effective Hulthén potential is a good approximation for the Yukawa potential as long as the value of $\alpha$ is not too high [31].

Using these approximations and considering $x=\mathrm{e}^{-2 \alpha r}$ and after some algebra we get the following differential equation:

$$
\begin{gathered}
{\left[-\frac{\epsilon}{4 \alpha^{2}} \frac{1}{x^{2}}+\frac{m V_{0}}{\alpha} \frac{1}{x(1-x)}-\frac{l(l+1)}{x(1-x)^{2}}\right] R_{n l}(x)} \\
+\frac{1}{x} \frac{\mathrm{d} R_{n l}(x)}{\mathrm{d} x}+\frac{\mathrm{d}^{2} R_{n l}(x)}{\mathrm{d} x^{2}}=0
\end{gathered}
$$

where $\epsilon=-2 m E$. To solve (14) using the Bethe ansatz method, the equation must be transformed in the form of (2). Therefore, let us assume that $R_{n l}(x)$ has the following form:

$$
R_{n l}(x)=x^{\sqrt{\epsilon} /(2 \alpha)}(1-x)^{l+1} Y(x) .
$$

In $(15)$, the function $Y(x)$ is dependent on $n$ with a non-trivial dependence. Substituting (15) in (14), we get

$$
\begin{aligned}
& {\left[\frac{l(l+1)}{(1-x)^{2}}-\frac{A}{(1-x) x^{2}}(l+x+2 l x)+\frac{A}{x^{2}}\right.} \\
& \left.\quad-\frac{l(l+1)}{(1-x)^{2}}+\frac{B-l(l+1)}{x}+\frac{B-l(l+1)}{1-x}\right] Y(x) \\
& \quad+\left(\frac{2 A+1}{x}-\frac{2(l+1)}{1-x}\right) Y^{\prime}(x)+Y^{\prime \prime}(x)=0 .
\end{aligned}
$$


After some algebra, we have

$$
\begin{aligned}
& {[(B-(2 A+1)(l+1)-l(l+1))(1-x)} \\
& \quad+(B-(2 A+1)(l+1)-l(l+1)) x] Y(x) \\
& \quad+[(2 A+1)(1-x)-2(l+1)] Y^{\prime}(x) \\
& \quad+x(1-x) Y^{\prime \prime}(x)=0,
\end{aligned}
$$

where $A=\frac{\sqrt{\epsilon}}{2 \alpha}$ and $B=\frac{m V_{0}}{\alpha}$. This differential equation is similar to (2) and therefore has a polynomial solution of degree $n=0,1,3, \ldots$ :

$$
Y(x)=\prod_{i=1}^{n}\left(x-x_{i}\right), \quad Y(x) \equiv 1 \quad \text { for } n=0,
$$

where $x_{i}$ s are the roots of the above polynomial satisfying (5)-(7) and determined by the Bethe ansatz (8). Therefore, we have

$$
\begin{aligned}
& a_{4}=a_{3}=a_{0}=0, \quad a_{2}=-1, \quad a_{1}=1 \\
& b_{3}=b_{2}=0, \quad b_{1}=-2(l+1)-2\left(\frac{\sqrt{\epsilon}}{2 \alpha}+\frac{1}{2}\right), \\
& b_{0}=-2\left(\frac{\sqrt{\epsilon}}{2 \alpha}+\frac{1}{2}\right), \\
& c_{2}=c_{1}=0, \\
& c_{0}=\frac{m V_{0}}{\alpha}-2\left(\frac{\sqrt{\epsilon}}{2 \alpha}+\frac{1}{2}\right)(l+1)-l(l+1) .
\end{aligned}
$$

Comparing these coefficients with those of $(5)-(7)$ and after some algebra the energy eigenvalues of the Yukawa potential are as follows:

$$
E_{n l}=-\frac{\alpha^{2}}{2 m}\left(\frac{\frac{m V_{0}}{\alpha}-(n+l+1)^{2}}{n+l+1}\right)^{2}
$$

where $n=0,1,2, \ldots$ and $l=0,1, \ldots$ are the integers satisfying the physical condition $n+l+1 \leq$ $\sqrt{\frac{m V_{0}}{\alpha}}$. We see that these eigenvalues are exactly similar to the formula obtained by Peña et al. [38], as well as NU [37], and BAM finds the energy spectra in an easier manner. In Table I, the numerical values of energy have been compared to the results of other works $[30,31,33-35]$ as well as the accurate variational method applied by Greene and Valdrich [30]. These results have been calculated for $\hbar=m=1, V_{0}=\sqrt{2}$ and different values of $g=\frac{\alpha}{V_{0}}$. As we can see from this table, the BAM results are in complete agreement with those of others, specially the NU method. This table shows that the differences in the present work and others (in some data) are very small and one can use BAM for the Yukawa potential with a good accuracy.

In Tables II and III, the results of the Bethe ansatz method have been presented for $\alpha=0.2 \mathrm{fm}^{-1}$ and $\hbar=2 m=1$ for $n=0$ and $n>0$, respectively, and the results have been compared with other calculations [33-35]. As it is clear from these tables, our results have a complete agreement with the NU method and generally agree with other analytical and numerical works. These tables imply that BAM can be used as a good quasi-exact method to find the ground state and excited states solutions of the wave equation with the Yukawa potential. From these tables, we can see that the energy of higher excited states differs slightly from BAM (as well as NU) at higher values of $V_{0}$. However, calculations show that the error is very small. To compare with [35] and [30], one should use $\frac{1}{g}=\frac{V_{0}}{\alpha}$ as the effective screening parameters $\left(Z D / a_{0}\right.$ in [35]). As it is mentioned in [35], in the limit of $g \rightarrow 0$ (alternatively $V_{0}$ in Tables II and III), the results reach those of numerical results in agreement with the current results. In the limit of $g \rightarrow \infty$ (small values of $Z D / a_{0}$ in [35] or high values of $V_{0}$ in these tables), the BAM results are slightly different from those of variational and exact numerical ones.

As we know, when $\alpha \rightarrow 0$, the Yukawa potential reduces to the Coulomb potential and therefore, the energy eigenvalues (22) must tend to the energy eigenvalues of a pure Coulomb interaction. Thus, after some algebra, in this limit we have

$$
E_{n l}=-\frac{m V_{0}^{2}}{2} \frac{1}{(n+l+1)^{2}}=-\frac{m V_{0}^{2}}{2} \frac{1}{{n^{\prime}}^{2}},
$$

where we set $n^{\prime}=n+l+1$. In (23), we have the energy eigenvalues of the Coulomb potential [39].

Now, in order to find the wave functions of the Yukawa potential, corresponding to $E_{n l}$, the roots in (18) (i.e., $\left.x_{i}\right)$ must be found. Therefore, in the case of $n=0$, we get

$$
Y(x)=1 \text {, }
$$

and

$$
R_{0 l}(x)=\left(1-\mathrm{e}^{-2 \alpha r}\right)^{l+1} \mathrm{e}^{-\sqrt{\epsilon} r} .
$$

For $n=1$, we have

$$
\left[-2(l+1)-2\left(\frac{\sqrt{\epsilon}}{2 \alpha}+\frac{1}{2}\right)\right] x_{1}-\left(2 \frac{\sqrt{\epsilon}}{2 \alpha}+1\right)=0,
$$

and therefore

$$
x_{1}=\frac{2\left(\frac{\sqrt{\epsilon}}{2 \alpha}+\frac{1}{2}\right)}{-2(l+1)-2\left(\frac{\sqrt{\epsilon}}{2 \alpha}+\frac{1}{2}\right)}
$$

and the wave function is as follows:

$$
\begin{aligned}
& R_{1 l}=\left(1-\mathrm{e}^{-2 \alpha r}\right)^{l+1} \mathrm{e}^{-\sqrt{\epsilon} r} \\
& \times\left(\mathrm{e}^{-2 \alpha r}-\frac{2 \frac{\sqrt{\epsilon}}{2 \alpha}+1}{-2(l+1)-2\left(\frac{\sqrt{\epsilon}}{2 \alpha}+\frac{1}{2}\right)}\right) .
\end{aligned}
$$

In the case of $n=2$, the following equations must be solved to find the roots:

$$
\begin{aligned}
& \frac{2}{x_{1}-x_{2}}+\frac{\left[-2(l+1)-2\left(\frac{\sqrt{\epsilon}}{2 \alpha}+\frac{1}{2}\right)\right] x_{1}}{x_{1}\left(1-x_{1}\right)} \\
& -\frac{2\left(\frac{\sqrt{\epsilon}}{2 \alpha}+\frac{1}{2}\right)}{x_{1}\left(1-x_{1}\right)}=0, \\
& \frac{2}{x_{2}-x_{1}}+\frac{\left[-2(l+1)-2\left(\frac{\sqrt{\epsilon}}{2 \alpha}+\frac{1}{2}\right)\right] x_{2}}{x_{2}\left(1-x_{2}\right)} \\
& -\frac{2\left(\frac{\sqrt{\epsilon}}{2 \alpha}+\frac{1}{2}\right)}{x_{2}\left(1-x_{2}\right)}=0,
\end{aligned}
$$


TABLE I

Some energy levels (in $\left[\mathrm{fm}^{-1}\right]$ ) of the Yukawa potential for different values of $g$ and comparison with other works. In this table, we set $\hbar=m=1$ and $V_{0}=\sqrt{2}$. ${ }^{*}$ The values of $s$-state energies have been deduced from the correspondence between Rogers et al. [35] and $l=0$ variational wave functions [31]

\begin{tabular}{|c|c|c|c|c|c|c|c|}
\hline State & $g$ & BAM & NU [37] & AIM [33] & SUSY [32] & Numerical [35] & Variational [30]* \\
\hline \multirow{6}{*}{$1 s$} & 0.002 & -0.99600 & -0.99600 & -0.99600 & -0.99601 & -0.99600 & -0.99600 \\
\hline & 0.005 & -0.99002 & -0.99002 & -0.99003 & -0.99004 & -0.99000 & -0.99090 \\
\hline & 0.010 & -0.98010 & -0.98010 & -0.98014 & -0.98015 & -0.98010 & -0.98010 \\
\hline & 0.020 & -0.96040 & -0.96040 & -0.96059 & -0.96059 & -0.96060 & -0.96060 \\
\hline & 0.025 & -0.95062 & -0.95062 & -0.95092 & -0.95092 & -0.95090 & -0.95090 \\
\hline & 0.050 & -0.90250 & -0.90250 & -0.90363 & -0.90363 & -0.90360 & -0.90360 \\
\hline \multirow{6}{*}{$2 s$} & 0.002 & -0.24601 & -0.24601 & -0.24602 & -0.24602 & -0.24600 & -0.24600 \\
\hline & 0.005 & -0.24010 & -0.24010 & -0.24014 & -0.24015 & -0.24010 & -0.24010 \\
\hline & 0.010 & -0.23040 & -0.23040 & -0.23058 & -0.23059 & -0.20360 & -0.20360 \\
\hline & 0.020 & -0.21160 & -0.21160 & -0.21229 & -0.21230 & -0.21230 & -0.21230 \\
\hline & 0.025 & -0.20250 & -0.20250 & -0.20355 & -0.20355 & -0.20360 & -0.20360 \\
\hline & 0.050 & -0.16000 & -0.16000 & -0.16354 & -0.16351 & -0.16350 & -0.16350 \\
\hline \multirow{6}{*}{$2 p$} & 0.002 & -0.24601 & -0.24601 & -0.24601 & -0.24602 & -0.24600 & - \\
\hline & 0.005 & -0.24010 & -0.24010 & -0.24012 & -0.24012 & -0.24010 & - \\
\hline & 0.010 & -0.23040 & -0.23040 & -0.23049 & -0.23049 & -0.23050 & -0.23050 \\
\hline & 0.020 & -0.21160 & -0.21160 & -0.21192 & -0.21192 & -0.21190 & -0.21190 \\
\hline & 0.025 & -0.20250 & -0.20250 & -0.20298 & -0.20299 & -0.20300 & -0.20300 \\
\hline & 0.050 & -0.16000 & -0.16000 & -0.16148 & -0.16144 & -0.16150 & -0.16150 \\
\hline \multirow{6}{*}{$3 p$} & 0.002 & -0.10715 & -0.10714 & -0.10716 & -0.10716 & -0.10720 & - \\
\hline & 0.005 & -0.10134 & -0.10133 & -0.10141 & -0.10142 & -0.10140 & - \\
\hline & 0.010 & -0.09201 & -0.09201 & -0.09230 & -0.09231 & -0.09231 & -0.09231 \\
\hline & 0.020 & -0.07471 & -0.07471 & -0.07570 & -0.07570 & -0.07570 & -0.07570 \\
\hline & 0.025 & -0.06674 & -0.06673 & -0.06815 & -0.06814 & -0.06816 & -0.06816 \\
\hline & 0.050 & -0.03361 & -0.03361 & -0.03711 & -0.03739 & -0.03712 & -0.03711 \\
\hline \multirow{6}{*}{$3 d$} & 0.002 & -0.10705 & -0.10714 & -0.10715 & -0.10715 & -0.10720 & - \\
\hline & 0.005 & -0.10133 & -0.10133 & -0.10136 & -0.1014 & -0.10140 & - \\
\hline & 0.010 & -0.09201 & -0.09201 & -0.09212 & -0.09212 & -0.09212 & -0.09212 \\
\hline & 0.020 & -0.07471 & -0.07471 & -0.07503 & -0.07502 & -0.07503 & -0.07503 \\
\hline & 0.025 & -0.06674 & -0.06673 & -0.06714 & -0.06713 & -0.06715 & -0.06715 \\
\hline & 0.050 & -0.03361 & -0.03361 & -0.03383 & -0.03388 & -0.03383 & -0.03383 \\
\hline
\end{tabular}

and therefore, the wave function can be written as

$$
\begin{aligned}
& R_{2 l}=\left(1-\mathrm{e}^{-2 \alpha r}\right)^{l+1} \mathrm{e}^{-\sqrt{\epsilon} r} \\
& \quad \times\left(\mathrm{e}^{-2 \alpha r}-x_{1}\right)\left(\mathrm{e}^{-2 \alpha r}-x_{2}\right),
\end{aligned}
$$

where $x_{1}$ and $x_{2}$ are the solutions of (29) and $\epsilon=-2 m E_{n l}$. For other values of $n$, one should solve (7) straightforwardly and the wave functions can be found using the roots of these equation for any state.

\section{Generalization to arbitrary dimension}

One of the advantages of BAM calculations in this paper is solving the $N$-dimensional problem. Based on the forepart discussions, a generalization to higher dimensions is very straightforward. To do this, let us consider the $n$-dimensional radial Schrödinger equation [40]:

$$
\begin{aligned}
& \frac{1}{r^{N-1}} \frac{\mathrm{d}}{\mathrm{d} r}\left(r^{N-1} \frac{\mathrm{d} u_{n l}(r)}{\mathrm{d} r}\right) \\
& +\frac{2 m}{\hbar^{2}}\left[E-\frac{l(l+N-2)}{r^{2}}-V(r)\right] u_{n l}(r)=0
\end{aligned}
$$

and using $u_{n l}(r)=\frac{R_{n l}(r)}{r}$ one can get the following equation:

$$
\begin{aligned}
& \frac{\mathrm{d}^{2} R_{n l}(r)}{\mathrm{d} r^{2}}+\frac{2 m}{\hbar^{2}}\left[E-V(r)-\frac{l(l+N-2)}{r^{2}}\right. \\
& \left.+\frac{(N-1)(N-3)}{4 r^{2}}\right] u_{n l}(r)=0 .
\end{aligned}
$$


TABLE II

The same description as in Table I, but these results have been computed for $\hbar=2 m=1, \alpha=0.2 \mathrm{fm}^{-1}$ and $n=0$ and different values of the screening length.

\begin{tabular}{c|c|c|c|c|c|c|c}
\hline \hline$V_{0}$ & $l$ & BAM & NU [37] & AIM [33] & SUSY [32] & Numerical [36] & Analytical [36] \\
\hline 4 & 0 & -3.24 & -3.24 & -3.2564 & -3.2563 & -3.2565 & -3.2199 \\
\hline \multirow{2}{*}{8} & 0 & -14.44 & -14.44 & -14.4581 & -14.4581 & -14.4571 & -14.4199 \\
& 1 & -2.56 & -2.56 & -2.5836 & -2.583 & -2.5836 & -2.4332 \\
\hline \multirow{2}{*}{16} & 0 & -60.84 & -60.84 & -60.859 & -60.859 & -60.859 & -60.8193 \\
& 1 & -12.96 & -12.96 & -12.991 & -12.9908 & -12.991 & -12.8375 \\
\hline \multirow{2}{*}{24} & 0 & -139.24 & -139.24 & -139.2593 & -139.259 & -139.2594 & -139.2201 \\
& 1 & -31.36 & -31.36 & -31.39381 & -31.3937 & -31.3938 & -31.2385 \\
& 2 & -11.56 & -11.56 & -11.5959 & -11.5951 & -11.5959 & -11.2456
\end{tabular}

The same description as in Table II, but $n>0$.

TABLE III

\begin{tabular}{c|c|c|c|c|c|c|c|c}
\hline \hline$V_{0}$ & $n$ & $l$ & BAM & NU [37] & AIM [33] & SUSY [32] & Numerical [36] & Analytical [36] \\
\hline \multirow{4}{*}{16} & 1 & 0 & -12.9600 & -12.9600 & -13.0273 & -13.0270 & -13.0273 & -13.0326 \\
& 2 & 0 & -4.2711 & -4.2711 & -4.3941 & -4.3937 & -4.3720 & -4.4057 \\
& 1 & 1 & -4.2711 & -4.2711 & -4.3621 & -4.3612 & -4.3480 & -4.3886 \\
\hline \multirow{6}{*}{24} & 1 & 0 & -31.3600 & -31.3600 & -31.4312 & -31.431 & -31.4356 & -31.4313 \\
& 2 & 0 & -11.5600 & -11.5600 & -11.6998 & -11.6990 & -1.6998 & -11.7093 \\
& 3 & 0 & -4.8400 & -4.8400 & -5.0441 & -5.0448 & -5.0442 & -5.0590 \\
& 4 & 0 & -1.9600 & -1.9600 & -2.2033 & -2.2194 & -2.2033 & -2.2237 \\
& 2 & 1 & -11.5600 & -11.5600 & -11.6652 & -11.6640 & -11.6653 & -11.6839 \\
& 2 & 1 & -4.8400 & -4.8400 & -5.0134 & -5.0133 & -5.0135 & -5.0541 \\
& 1 & 1 & -1.9600 & -1.9600 & -2.1770 & -2.1908 & -2.1770 & -2.2414 \\
& 2 & 2 & -4.8400 & -4.8400 & -4.9515 & -4.9504 & -4.9516 & -5.0085 \\
& 2 & 2 & -1.9600 & -1.9600 & -2.1241 & -2.1337 & -2.1241 & -2.2428
\end{tabular}

Now, using the same procedure mentioned as in (11)-(14), we get the following equation:

$$
\begin{aligned}
& \frac{\mathrm{d}^{2} R_{n l}(x)}{\mathrm{d} x^{2}}+\frac{1}{x} \frac{\mathrm{d} R_{n l}(x)}{\mathrm{d} x}+\left(-\frac{\epsilon / 4 \alpha^{2}}{x^{2}}+\frac{m V_{0} / \alpha}{x(1-x)}\right. \\
& \left.-\frac{l(l+N-2)+\frac{1}{4}(N-1)(N-3)}{x(1-x)^{2}}\right) R_{n l}(x)=0,
\end{aligned}
$$

which, except for the last term in brackets, is the same as (14). Therefore, one can try the following idea for the wave function:

$$
R_{n l}(x)=x^{\sqrt{\epsilon} /(2 \alpha)}(1-x)^{c_{1}} Y(x) .
$$

After some algebra, in the sense of the BAM equations mentioned in the previous sections, the constant $c_{1}$ must satisfy the following equation:

$$
c_{1}\left(c_{1}-1\right)=l(l+1)+l\left(l+\frac{1}{4}(N-1)(N-3)\right) .
$$

After finding $c_{1}$ one can find all the roots of the BAM equations as well as wave functions. It is clear that in three dimensions, $N=3$, we have $c_{1}=l+1$ and one gets the previous results.

\section{Conclusion}

One of the quasi-exact solution methods to solve the Schrödinger equation is the Bethe ansatz method based on polynomial expansion of solutions. Because of this and because of the importance of the Yukawa potential in physics, in this paper BAM has been used to find energy eigenvalues and eigenfunctions of the Schrödinger equation. The results showed a good agreement with other works and this method can be used as an accurate method in this problem. In the case of a zero screening parameter limit, the eigenvalues tend to that of the Coulomb potential. The difference between the applied method and other methods investigated is that at higher levels the BAM method is slightly less accurate for higher values of $V_{0}$ (potential strength). But, the difference is minor and the BAM method is a reliable method to find the solution of Yukawatype potentials. It is found that, based on the results of the three-dimensional radial equation, the generalization to other dimensions is very straightforward. 


\section{Acknowledgments}

Financial support from the Ilam University Research Council is gratefully acknowledged.

\section{References}

[1] S.H. Dong, Commun. Theor. Phys. 55, 969 (2011).

[2] C.S. Jia, P. Gao, X.L. Peng, J. Phys. A 39, 7737 (2006).

[3] X. Zou, L.Z. Yi, C.S. Jia, Phys. Lett. A 346, 54 (2005).

[4] Y.F. Diao, L.Z. Yi, T. Chen, C.S. Jia, Mod. Phys. Lett. B 23, 2269 (2009).

[5] C. Berkdemir, A. Berkdemir, R. Sever, Phys. Rev. C 74, 039902 (2006).

[6] A. Arda, R. Sever, Int. J. Mod. Phys. C 19, 763 (2008).

[7] X.Y. Chen, T. Chen, C.S. Jia, Eur. Phys. J. Plus 129, 75 (2014).

[8] C.S. Jia, J.W. Dai, L.H. Zhang, J.Y. Liu, X.L. Peng, Phys. Lett. A 379, 137 (2015).

[9] S.M. Ikhdair, ISRN-Math. Phys. 2012, 201525 (2012).

[10] S.M. Ikhdair, Phys. Scr. 83, 015010 (2011).

[11] M.G. Miranda, G. Sun, S.H. Dong, Int. J. Mod. Phys. E 129, 123 (2010).

[12] S.M. Ikhdair, B.J. Falaye, Chem. Phys. 421, 84 (2013).

[13] H. Motavali, Mod. Phys. Lett. A 24, 1227 (2009).

[14] G.F. Wei, X.Y. Duan, X.Y. Liu, Int. J. Mod. Phys. A 25, 1649 (2010).

[15] S.M. Ikhdair, R. Sever, Phys. Scr. 79, 035002 (2009).

[16] S.M. Ikhdair, C. Berkdemir, R. Sever, Appl. Math. Comput. 217, 9019 (2011).

[17] F. Cooper, A. Khare, U. Sukhatme, Phys. Rep. C 251, 267 (1995).

[18] X.C. Zhang, Q.W. Liu, C.S. Jia, L.Z. Wang, Phys. Lett. A 340, 59 (2005).

[19] E. Delabaere, F. Bham, Phys. Lett. A 250, 25 (1998).
[20] C.M. Bender, K.A. Milton, V.M. Savage, Phys. Rev. D 62, 085001 (2000).

[21] N. Hatamia, J. Naji, M. Pananeh, Eur. Phys. J. Plus 134, 90 (2019).

[22] D. Gomez-Ullate, N. Kamran, R. Milson, J. Math. Anal. Appl. 359, 352 (2009).

[23] B. Shapiro, M. Tater, arXiv:0812.2321v1 (2008), arXiv:0904.0650v1 (2009).

[24] C.M. Bender, G.V. Dunne, J. Math. Phys. 37, 6 (1996).

[25] Y.Z. Zhong, J. Phys. A Math. Theor. 45, 065206 (2012).

[26] H. Yukawa, Proc. Phys. Math. Soc. Jpn. 17, 48 (1935).

[27] J. McEnnan, L. Kissel, R.H. Pratt, Phys. Rev. A 13, 532 (1976).

[28] C.H. Mehta, S.H. Patil, Phys. Rev. A 17, 34 (1978).

[29] R. Dutt, Y.P. Varshni, Z. Phys. A 313 , 143 (1983).

[30] R.L. Greene, C. Aldrich, Phys. Rev. A 14, 2363 (1976).

[31] C.S. Lam, Y.P. Varshni, Phys. Rev. A 4, 1875 (1971).

[32] B. Gonul, K. Koksal, E. Bakir, Phys. Scr. 73, 279 (2006).

[33] M. Karakoc, J. Boztosun, Int. J. Mod. Phys. E 15, 1253 (2006).

[34] E.Z. Liverts, E.G. Drukarev, R. Krivec, V.B. Mandelzweig, Few Body Syst. 44, 367 (2008).

[35] F.J. Rogers, H.C. Graboske, D.J. Harwood, Phys. Rev. A 1, 1577 (1970).

[36] B. Chakrabarti, T.K. Das, Phys. Lett. A 285, 11 (2001).

[37] M. Hamzavi, M. Movahedi, K.-E. Thylwe, A.A. Rajabi, Chin. Phys. Lett. 29, 080302 (2012).

[38] J.J. Peña, J. Morales, J. García-Martínez, J. García-Ravelo, Mol. Phys. 113, 260 (2015).

[39] L.I. Schiff, Quantum Mechanics, 3rd ed., McGraw-Hill, New York 1955.

[40] H. Hassanabadi, L.L. Lu, S. Zarrinkamar, G.H. Liu, H. Rahimov, Acta Phys. Pol. A 122, 650 (2012). 